\title{
AC 2008-686: FRESHMAN PROJECT: DISCOVERING GLOBAL TRENDS - A SURVEY OF THE ENVIRONMENTAL STRATEGIES OF THE AEROSPACE INDUSTRY
}

\author{
Alexander Friess, Dubai Aerospace Enterprise University \\ Alexander Friess is an Assistant Professor of Engineering at Dubai Aerospace Enterprise \\ University. He received his B.Sc. in Physics and M.Sc. and Ph.D. in Aeronautical Engineering \\ from Rensselaer Polytechnic Institute. His research background includes experimental fluid \\ dynamics, composite materials and performance optimization, and he has been active globally as \\ consultant and design engineer working on a variety of projects, including participating in the \\ design and engineering of South Africa's yacht for the America's Cup 2007. Current interests \\ include instrumentation, renewable energy applications, and the field of engineering design (i.e. \\ design innovation, inclusiveness and usability), as well as design education with a focus on \\ multinational student collaboration and educating the "global engineer".
}

\section{Carol Briam, Dubai Aerospace Enterprise University}

Carol Briam is an Assistant Professor of English at Dubai Aerospace Enterprise University. She received a Ph.D. in English Rhetoric and Linguistics from Indiana University of Pennsylvania and an MBA from Thunderbird School of Global Management. She also holds master's and bachelor's degrees from the University of Arizona. A former U.S. Foreign Service Officer, she lived in Africa, Asia, Europe, and South America, before moving to Dubai in 2007. Her interests lie in the areas of technical and management communication, information design, and cross-cultural communication.

\section{Linda Thompson, Dubai Aerospace Enterprise University}

Linda Thompson is Director of Integrated Curriculum at Dubai Aerospace Enterprise University. She teaches the University Success class for all first year students. She received her B.A. in Elementary Education, M.S. in Curriculum and Instruction and Ph.D. in Educational Administration from Purdue University, West Lafayette, Indiana, USA. Her research interests include administrative leadership for diverse populations, increasing ESL student achievement, and meeting the needs of secondary ESL students in the content areas. Current interests include, developing programs which ensure university success for ESL students, integrated curriculum at an aviation focused university, and accreditation processes.

\section{Hemdeep Dulthummon, Dubai Aerospace Enterprise University}

Hemdeep Dulthummon is an undergraduate student at Dubai Aerospace Enterprise University. He is currently doing a B.Sc. in Flight and Aviation Management but plans to switch to Aerospace Engineering. 


\section{Freshman Project: Discovering Global Trends - a Survey of Environmental Strategies of the Aerospace Industry}

\section{Short Abstract}

A first semester student project that surveys the initiatives of the commercial aerospace industry to address increasing global environmental concerns is presented here. The project motivation stems from a twofold need for students of the region, and for all participants (who were non-native English speakers): (1) to start developing at an early stage the process skills required to successfully operate in the global environment of the aerospace industry, and (2) to gain exposure to the growing importance of environmental concerns within the industry, both from a technical and a business perspective.

The students were organized in multinational teams that in the context of the 2007 Dubai Air Show explored different branches of the industry with regards to environmental policies and strategies. These branches included the airlines, airports, manufacturers, and regulatory agencies. In addition, a series of workshops on research skills, presentation skills, professional communication and behavior, were offered to prepare the students both for a successful experience at the Air Show and to assist them in presenting their findings in a closing presentation to their peers, a faculty panel, and industry representatives.

The results assessment was initially constructed on the assumption that the student motivation to participate in the project would be strong, because a portion of the grade for the course Introduction to Aviation would depend on student performance on the project. In addition, the winning team would receive introductory flights in the university's flight academy aircraft. The individual assessment of the twofold learning objectives, technical content and process skills, was to be carried out on an exclusive team based grade, as assessed by an expert panel composed of faculty, staff and industry representatives during the closing presentation. However, this assumption, in the course of the formative course assessment, proved to be erroneous, and the grading scheme, in order to retain fairness, was changed to a more complex system, composed of both team and individual assessments as well as a team participation via a peer survey.

\section{Introduction}

The civilian aerospace industry is, by definition, one that creates products and services that allow the rapid, safe, and cost-effective worldwide movement of people and goods. Although cyclical in nature and closely linked to the condition of the global economy, the industry has experienced sustained growth rates over the past decade, most notably in the developing eastern economies, where annual fleet growth has reached double digit figures.

Announcements of new aircraft purchases chase each other in the region, and the manufacturers are more than ever looking eastward for their primary market expansion.

This rapid global growth requires an increasing supply of aviation professionals in all branches of the industry, and further requires them to be comfortable in working and communicating with their peers who may well be of different national origins. These considerations are of special interest in the Middle East, where cultural diversity is a fact of life, and where English is often spoken as a second language. 
Thus, any university offering aviation-oriented programs in this part of the world has an obligation to give students opportunities to practice intercultural teamwork and communication. Finding such opportunities is relatively straightforward at Dubai Aerospace Enterprise University in that the university is located at the geographic focal point of eastwards expansion, and has direct access to the Dubai Air Show, one of the leading international stages for the aerospace industry. This year, orders at the Air Show have beaten all sales records, mostly from Middle Eastern carriers.

In recent years there has been a surge of activity to expose university students to international experiences ${ }^{1}$. The increasing awareness that, in addition to all technical knowhow, the process skills, and more specifically the international process skills, are becoming more and more important in today's globalized world, have coined terms such as global competencies and global engineer ${ }^{2,3}$, which are nowadays the buzzwords in higher education. While this trend is highly desirable in US and European academic environments, it is indispensable in the Middle East ${ }^{4}$, as there is no alternative to interacting with other, often very different nationalities and cultures on a daily basis. The region is experiencing unprecedented growth, and the limitation for more rapid expansion is skilled personnel capable of acting on a global stage. This need is a primary trigger for DAEU's mission, and thus projects such as the one presented here instill a strong international component from the very beginning of a student's studies at Dubai Aerospace Enterprise University.

The students of Dubai Aerospace Enterprise University defy categorization. Constituting the first freshman class of this new university, students are drawn from the United Arab Emirates, as well as other countries in the Middle East, South Asia, and Africa. Among some 20 students, at least 12 different countries are represented. Moreover, most students have more than one country affiliation, being nationals of one country and residents of another. For example, about one-third of the students are Indian citizens who reside in the UAE. A number of students have several country affiliations due to the diverging nationalities of their mother and father. School backgrounds are just as diverse as students' national identities. Students' high school degrees represent various national or international systems, including American, British, Emirati, Indian, and the Geneva-based International Baccalaureate.

This diversity, while enriching for students and faculty alike, potentially complicates team dynamics for a project such as this one. Students must deal not only with different personalities, but also with different cultural backgrounds and school experiences involving teams. Some high school experiences, for example, cause students to harbor notions of teams as being fully controlled and guided by teachers, with no allowance for team autonomy and decision-making.

Logistical factors also complicate team operations. Because no on-campus housing exists, most students commute to the university from diverse locations in Dubai, which is wellknown for its traffic bottlenecks. Several students have longer commutes from neighboring cities that are several hours away by car or bus. Thus, evening or weekend meeting times for teams are not feasible, with all team planning occurring, by necessity, during the day between classes. Student teams, wherever they are, tend to struggle when collaborating on presentations ${ }^{5}$. Conflicts arise due to such issues as personality clashes, unequal work contributions, and inability to reach consensus ${ }^{5}$. When such conflicts arise among teams that are relatively homogenous in terms of cultural and educational background, it seems inevitable that problems will arise when teams are made up of students representing diverse cultures and educational systems. 


\section{The Project:}

The formal announcement of the student project took place about one month before the actual Air Show. During an Introduction to Aviation class, students were presented with the framework of the project. The framework included a schedule of events that would prepare them for their interactions at the Air Show, recommendations for teamwork and cooperation strategies, and a presentation of the available support structure. This support structure consisted of an advisory panel, assembled from the University's staff and faculty, and including high-ranking industry representatives. The formal events that would take place before the air show included a workshop on background research, and a workshop on communication and interaction with the professionals at the show.

Four groups of students were assigned according to four broad themes: airlines, airports, governing agencies, and aircraft manufacturers. The mission statement was to research these branches and assess their respective efforts in addressing environmental considerations in their daily operation and strategic planning.

Attending the Dubai Air Show was undoubtedly a heady event for the students. As members of the first class of the university, students were treated like VIPs during the Air Show. They also were excused from classes for two days during the week of the big event, which featured 900 exhibits from some 50 countries.

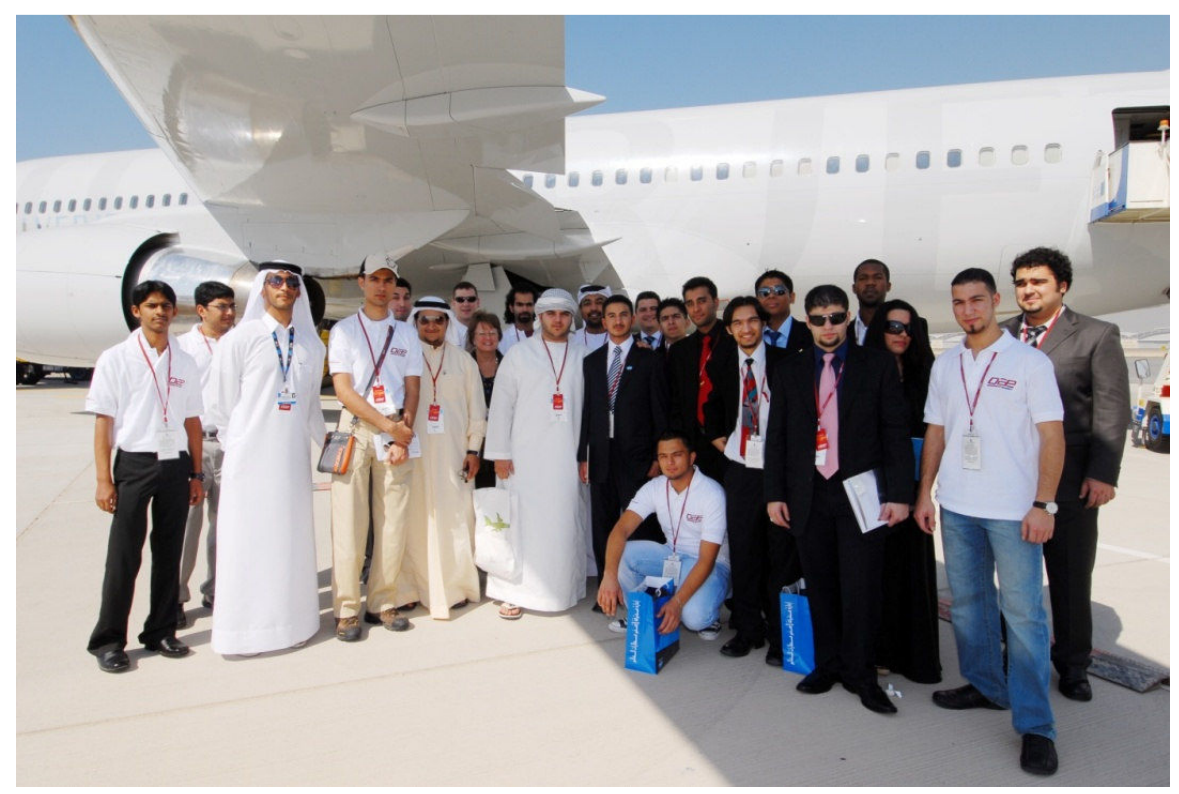

Figure 1. DAEU students at the Dubai Airshow

During the week that followed the Air Show, it was time for the students to "come back down to earth" and attend organized presentation seminars. While it was assumed that the entire class would attend two presentation development seminars, in preparation for the actual presentations, scant attendance at the first of these seminars was the rude reality. The organizer of the seminars later heard that some of the students who had not attended believed that attending such seminars would be "childish." Because some students had had a public speaking class in high school, they apparently assumed that their oral presentation education was complete, not realizing that honing presentation skills is-or should be-an ongoing 
quest. In contrast to the low attendance at the first seminar, attendance improved at the following week's presentation seminar. The fact that the actual presentation loomed near in the future (the following week) probably was the key motivating factor.

The day of the big event, a nervous buzz reigned among the students. The actual presentations were held in an auditorium, and a university-wide announcement had been sent out inviting all staff and faculty to attend. Specific topics researched and presented by the student teams included: noise and chemical pollution, use of renewable energies sources for airports and ground vehicles, the utilization of bio-fuels by the airlines, emissions trading and emission related landing fees at airports, use of modern materials in aircraft construction to maximize fuel efficiency, etc ... During the presentations, students were rated on content and delivery by 10 panelists, made up of industry representatives, staff members, and faculty members. On-the-spot rankings were compiled in an Excel spreadsheet.

Following the afternoon event, which lasted about three hours, the top four winners were announced at an outdoor barbecue celebration. A show-of-hands later that evening revealed that for most of the students, the afternoon's presentation represented their first such experience. Whatever shortcomings faculty may have noticed in the students' presentations, the fact that most were first-time presenters - presenting in a non-native language certainly kept their performance in perspective.

\section{Results assessment}

The results assessment can be divided into formative and summative assessment segments ${ }^{6}$ for both project evaluation and student performance outcome. While, and due to the short duration of the project and the envisioned direct interaction with the advisory panel, no discrete formative content and structure evaluations were set up, this informal interaction proved to be an effective feedback mechanism that triggered significant changes in the project. The summative assessment was carried out in the form of a post-project student survey $^{7}$ and a faculty and advisory panel debriefing.

\section{Formative assessment}

The formative assessment came about through student-faculty interactions, both in the workshops and in conversations with students. This "informal" formative evaluation, during the course of the project, led to the conclusion that the original team grading system was not appropriate, as unforeseen developments were skewing its fairness. From the outset, a number of students were not attending the compulsory workshops. In addition, these same students were not participating on their teams. Their concerned team members were worried that this situation would hurt their overall team grade.

The initial design of the grading system was based on the assumption that students wanted more than to earn a good grade. The assumption was that they were enthusiastic about aviation and poised to compete for the top prize : training flights in the University's Flight Academy aircraft for the winning team. Overlooked was the fact that a relative lack of team spirit and motivation on the part of a small fraction of the students was highly de-motivating to the other students. This behavior by a minority of the students continued even after repeated warnings of the faculty and advisory panel. 
In light of developments, the initial "team grade only" criterion was changed to "individual grades" for each student to reward their individual contributions. The resulting grading system included an assessment of both the individual and the team performance. This assessment was carried out during the presentations by a panel of 10 faculty, staff and industry representatives. In addition, the teams internally evaluated the contribution of each team member to the overall team effort by means of a previously carried out peer assessment. This peer assessment (termed Team Contribution) effectively provided the tool to distribute the panel's team grade over the team constituents, allocating more team credit to the students who had worked harder. The resulting overall grade for each student was composed of the sum of both the individual grade and the team grade (as distributed by the peer assessment), where the team grade was weighted at $25 \%$ of the overall grade, and the individual grade at $75 \%$. In addition, a passing "team contribution" grade had to be reached to be allowed to present as part of a team. If this team contribution grade was failed, the student would lose the team portion of the grade, and not be allowed to present as part of their team, but rather only as an individual. While this change in the grading did not meet general enthusiasm, especially by the students who were relying on their peers' efforts, it was welcome by the majority of the class. The final results are indicative of the success of this arrangement, as the winners clearly reflect the students who have excelled in their team environment, as well as in their individual technical research efforts.

\section{Summative assessment}

The summative project assessment took the form of a faculty and advisory panel debriefing, and a student survey evaluating their impressions.

\section{Student surveys}

The following charts depict the student survey results when grouped under the respective headings, which correspond to the desired learning objectives of process skills and technical industry knowledge (in addition to an instructor and workshop effectiveness assessment).

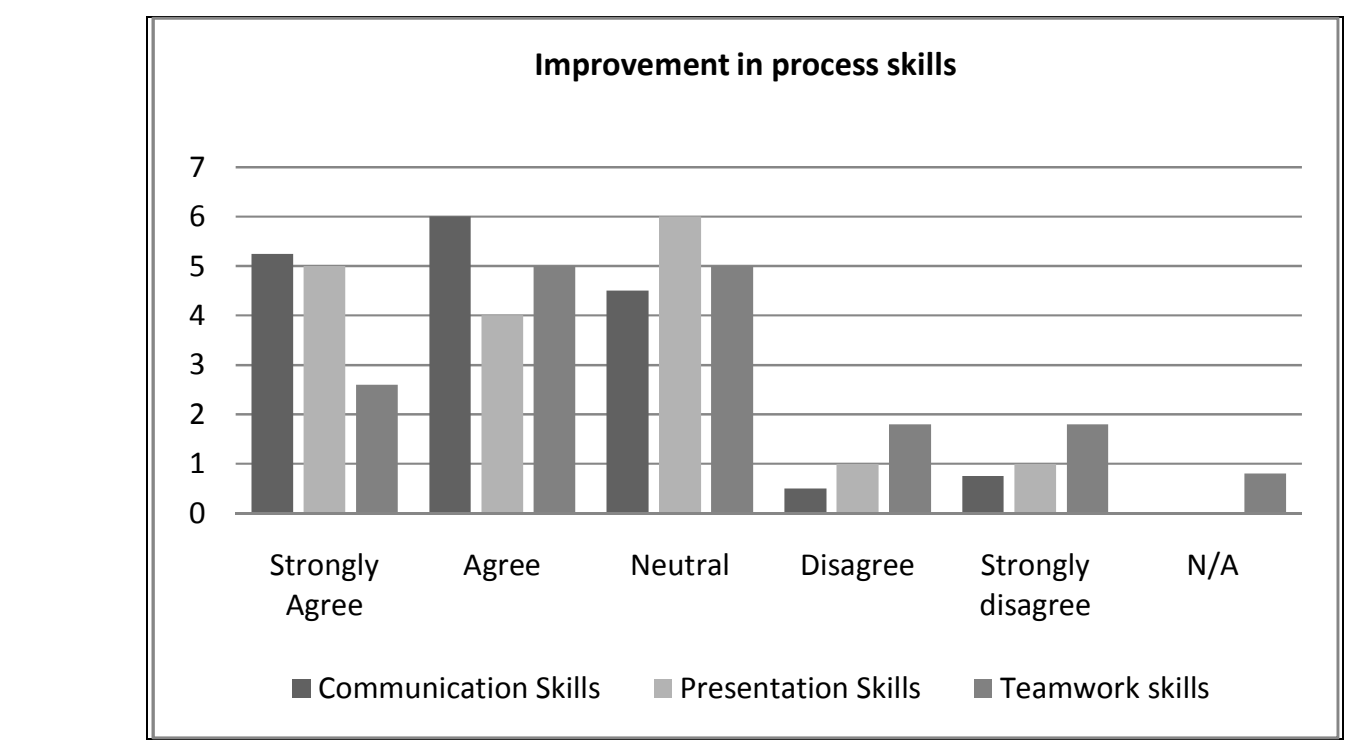

Figure 2. Student survey results - perceived improvement in process skills

The achievement of the process skills learning objective was widely assessed as positive. The Air Show gave ample opportunity to the students to practice their professional 
communication skills at an interpersonal and cross cultural level, and their confidence in these communication skills was also boosted. This heading combines survey statements such as "I have had to communicate with people from other countries", and "I feel more confident in my professional communication skills", and "I am more confident in my ability to interact with professionals in the field".

The team presentations were a display of strong verbal and presentation skills. This heading was composed of survey statements such as "I feel more confident in my oral presentation skills", and "I feel more confident in the use of technology during presentations", and clearly underlines the predominant verbal character of the local culture. The available presentation technology was used effectively, and the overall presentation event was perceived as a success. As the level of English and the personal confidence in presenting go hand in hand, the project lead a significant portion of the participating students to become members of Toastmasters to hone their public speaking skills, which by itself can be considered a success in encouraging life-long learning. Survey results clearly show a perceived improvement of the student's confidence and ability in presenting in front of a professional audience.

Teamwork skills (and these include survey statements such as "Teamwork Skills were practiced" and "I have improved my teamwork Skills", and "I have improved my time management skills"), albeit still perceived as positive, clearly show a more moderated enthusiasm. This is caused by the difficulties that were encountered, and that triggered a reform of the student grading system.

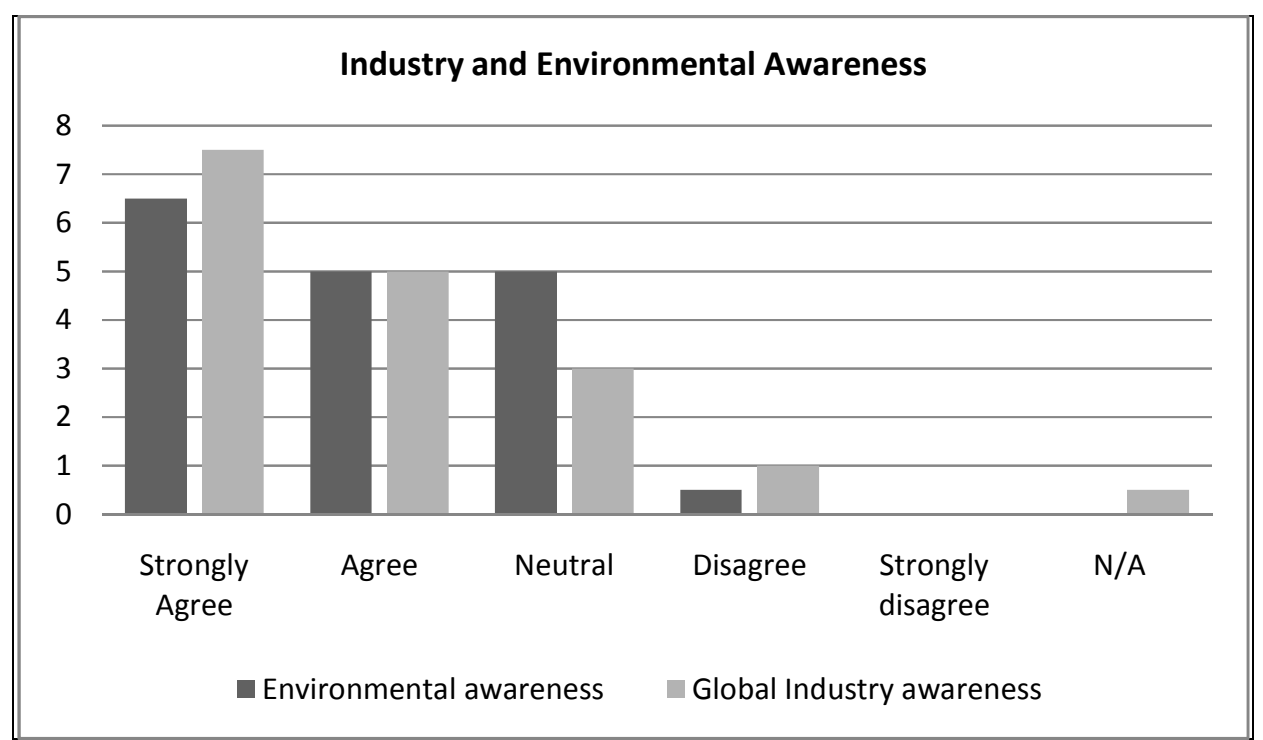

Figure 3. Student survey results on perceived improvements in industry understanding and related environmental considerations.

The learning objective of gaining an understanding of the industry, its global nature, and its increasing environmental pressures, was perceived as highly successful. The vendor and professional interaction of the students at the Air Show generated a completely new understanding of their chosen career, and reinforced and motivated them for their academic challenges. A project such as this, where first year students experience firsthand the professional environment of their chosen field of study, has generated beneficial repercussions from a variety of viewpoints, including a very low first-year drop-out rate. The 
group of 21 students that participated in this project, now in semester two, has only seen one student drop out.

The technical content researched and presented varied from rather superficial (last minute) analysis of the environmental actions of the different branches of the aerospace industry, to thoroughly investigated accounts of industry actions and facts. A difficulty was that each team was assigned a different branch of the industry, and not all had the same amount of material readily available. In addition, there is also overlap between the branches, and thus some of the material presented was repeated by more than one group. However, the student's perception of having significantly expanded their knowledge in the technicalities of environmental awareness and policy is clearly demonstrated in the survey results.

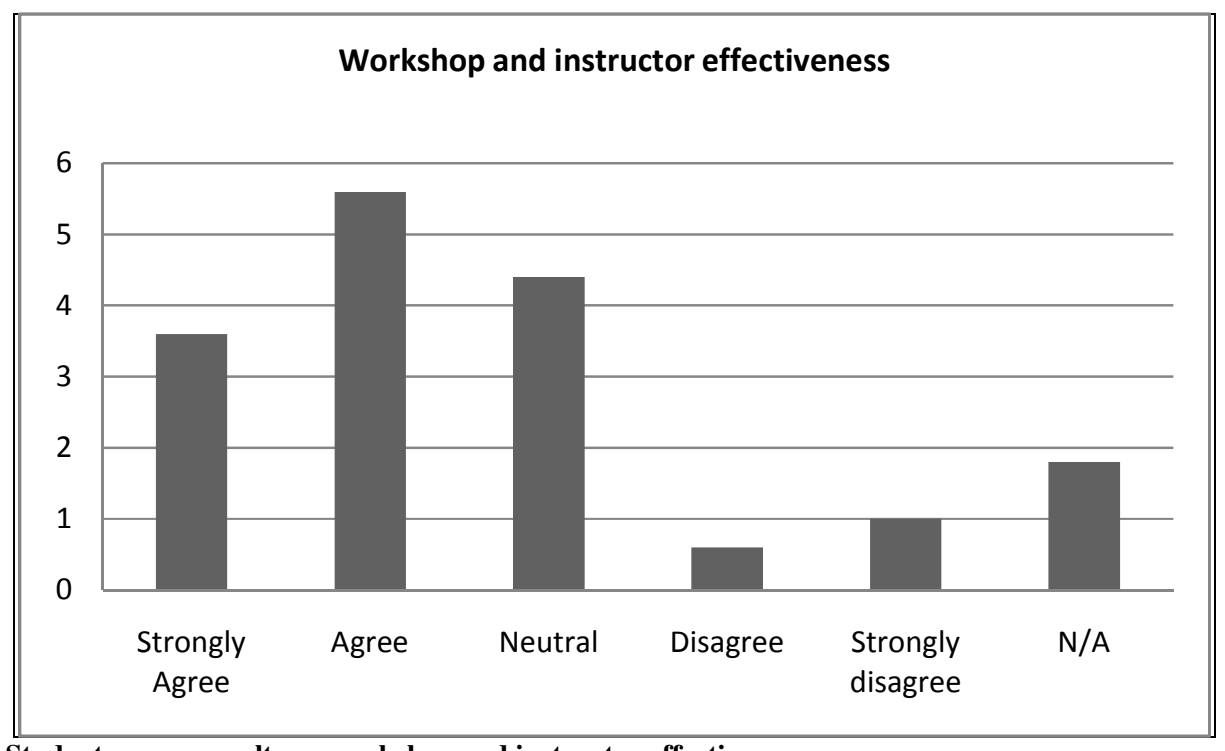

Figure 4. Student survey results on workshop and instructor effectiveness

The last heading on the student survey represents the student's perception of the instructor and workshop effectiveness. Survey statements such as "the instructors were helpful in the course of the project", "I have profited from the presentation workshop", "I have profited from the research workshop" constitute the basis for the results depicted in figure 4.

The current small class size allows a very high level of student-faculty interaction, which in turn also facilitated the timely recognition of the teamwork difficulties in the formative phase. However, and precisely because of the small inaugural class size, the students are used to this high level of interaction. This "habit" may in turn have represented a hindrance in stimulating teamwork activities on an independent basis. Figure 4 shows that, albeit a great majority of the students found the workshops and instructor interaction useful, also a small portion of the student body expressed discontent. The overall workshop attendance was not satisfactory, and the instructor pressure to make the students attend was initially not well received. However, and in light of the results of Figure 4, and in hindsight, the students appreciated the effectiveness of these workshops.

\section{The panel debriefing}

The debriefing took place among the faculty members that organized the project and the advisory panel members a few days after the team presentation. Major conclusions were that the students had made significant progress in their presentation and communication skills. This impression was ratified by the comments of some industry representatives that only had 
contact with the student at the beginning of the project, and then again during the presentations. Their distance to the day to day of the project proved highly valuable in assessing the progress of the students.

The overall impression of the technical soundness of the research presented varied from group to group. Some, such as the airplane manufacturer's group, achieved good technical content, exploring issues such as bio-fuels, hush-kits, fuel efficient high bypass ratio turbofans, and the next airliner generation made out of composite materials. Others, such as the regulatory agencies group, and partly due to the lesser amount of publically available information, struggled to attain the required depth. It was however perceived by the faculty (and ratified by the student survey) that significant progress was made in understanding the nature of the industry, and that the students gained substantial insight into the environmental concerns affecting the industry.

From a motivational standpoint it was deemed a highly successful project. The interaction with the industry professionals at the Air Show was a highlight for the students. The strategy to make them feel as part of the industry as opposed to mere observers paid off in dedication and motivation.

The panel also agreed that the varied teamwork skills and previous experience represented the Achilles' heel of the project. It had become clear early on that the students' previous teamwork experiences ranged from none at all to US high school level exposure. This imbalance in teamwork skills was not accounted for in the initial project design, and was even aggravated by inherent cultural differences of the different nationalities of the student body. While these difficulties emerged, it was also agreed that the formative assessment had worked, and that the corrective measures taken (the changing of the grading system) were, while unfortunate, also necessary and adequate. The principal lesson learned was that the teamwork outcome will require more foundational work in future projects, such as dedicated preparatory teamwork workshops.

\section{Conclusion}

The somewhat special nature of the project, only being about 6 weeks long, in combination with the diverse background of our students, and the multinational environment offered by the location of the university, have resulted in a very challenging and interesting learning experience for everybody involved. The learning objectives were defined to enhance the students' process skills and global awareness and competencies, and to enhance their

knowledge of the aerospace industry and current industry developments and concerns. These objectives arose because of the clear need to break down the communication barriers that are often encountered in global industries, and that are especially prominent between the eastern and western cultures. The location of the University, in Dubai, places it at a focal point between the east and the west, and current globalization trends and eastward market expansion of the western driven aerospace industry make the Dubai Air Show a truly global marketplace. The students of the region, perhaps more so than their western counterparts, need to attain global competence, and are thus involved from the very first semester in projects (such as this one) aimed at enhancing these skills.

The Air Show project has been an excellent showcase of the global nature of the industry to our students. The learning objectives have been achieved to a large extent. They (as judged both by them and by the assessment panel) have improved their presentation skills, they feel 
more comfortable in professional communication, they have had a chance to experience cross cultural communication, they are more aware of the global nature of the industry, and they feel that they have a better overview of their chosen profession. From a technical standpoint, the students have investigated the current trends in environmental awareness, global warming, and alternative energies as applicable to the aerospace industry, and have a higher confidence in tackling problems of a technical nature. At the same time, the diversity in backgrounds and mentalities have generated unforeseen difficulties in the homogeneity of the motivation and teamwork standards of the student body; specially the teamwork aspect was a difficult one, and is a recurring theme in the very hierarchical and tribal middle eastern culture. Lessons learned revolve around the need for an increased focus on more direct guidance of the team activities (much more so than what would be required in a US university). While the project still remains open ended, there is a need to structure the teamwork interaction and to guide time management, at least for the first stages of the project.

\section{Acknowledgements:}

The authors wish to thank Dubai Aerospace Enterprise University for providing the necessary funding for the project, the integrands of the advisory Panel for their efforts and support, and the many industry professionals who, through their interaction with our students, have shared the very essence of the industry with the next generation.

\section{Bibliography}

1. Esparragoza, I.E., Larrondo-Petrie, M., Jordan, R., and Paez, J. Forming the Global Engineer for the Americas: Global Educational Experiences and Opportunities Involving Latin America and the Caribbean. Proceeding of the 2007 ASEE Annual Conference and Exhibition, Honolulu, Hawaii June 24 to 27, 2007, AC 2007-576.

2. Downey, G.L., Lucena, J.C., Moskal, B.M., Parkhurst, R. et. Al. The Globally Competent Engineer: Working effectively with People Who Define Problems Differently. Journal of Engineering Education; April 2006; 95,2.

3. Hunter, W.D., Got Global Competency?. International Educator, Spring 2004; 13,2.

4. Adapting Engineering Design Model to Middle Eastern Culture: The Colorado School of Mines Brings Engineering Design to the Petroleum Institute. Scott, S.W., Proceedings of the 2005 ASEE Annual Conference \& Exposition.

5. Applying Project Management Strategies to Collaborative Projects in Business Communication and Writing Courses. Andersen, R., Proceedings of the 2005 Association for Business Communication Annual Convention.

6. NSF 02-057, The 2002 User-Friendly Handbook for Project Evaluation. http://www.nsf.gov/pubs/2002/nsf02057/start.htm

7. Suskie, Linda. Questionnaire Survey Research, What Works. $2^{\text {nd }}$ Ed. Association for Institutional Research. 